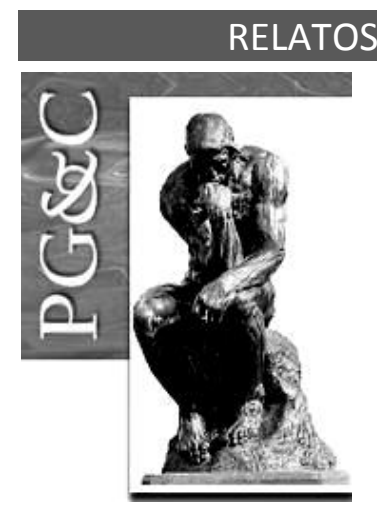

\title{
COMPETÊNCIAS GERENCIAIS: UMA PERSPECTIVA DOS GESTORES DAS EMPRESAS DO AGRONEGÓCIO
}

\author{
Lidiane Cássia Comin \\ Mestre em Administração pela Faculdade Meridional de Passo Fundo, \\ Brasil. Professora da Faculdade FAT-Faculdade e Escola, Tapejara, Brasil. \\ E-mail: Ic.comin@bol.com.br

\section{Eliana Andrea Severo} \\ Doutora em Administração pela Pontifícia Universidade Católica do Rio \\ Grande do Sul, Brasil. Professora da Faculdade Meridional de Passo Fundo, \\ Brasil. \\ E-mail: eliana.severo@imed.edu.br
}

\section{Camila Fortuna Dall Agnol}

Mestre em Administração pela Faculdade Meridional de Passo Fundo, Brasil.

E-mail: camila.parceria@terra.com.br

Luciane Soares de Medeiros

Mestre em Administração pela Faculdade Meridional de Passo Fundo, Brasil. Professor da Universidade Luterana do Brasil, Carazinho, Brasil. E-mail: luciane medeiros@yahoo.com.br

\section{Julio Cesar Ferro de Guimarães}

Doutor em Administração pela Pontifícia Universidade Católica do Rio Grande do Sul, Brasil. Professor da Universidade Federal de Pelotas, Brasil.

E-mail: juliocfguimaraes@yahoo.com.br

\section{Resumo}

O objetivo deste estudo é identificar as competências gerenciais sob a percepção dos gestores em suas tomadas de decisões. A fundamentação teórica apresenta uma breve discussão dos conceitos de competências que auxiliam no entendimento do estudo. A coleta dos dados foi realizada com 149 gestores de empresas que atuam no setor do agronegócio, a técnica utilizada para análise destes dados foi a análise fatorial exploratória, sendo possível a extração de seis fatores principais que agruparam as variáveis do estudo de forma diferenciada ao modelo inicial do estudo proposto, criando assim uma nova escala estatística que pode ser utilizada em futuras investigações empíricas para mensurar as competências gerencias em diferentes contextos de estudo. Corroborando com a literatura, o estudo apresenta um framework identificando seis dimensões pelas quais os gestores percebem e reconhecem as competências necessárias no processo de gestão das empresas do agronegócio. Assim, conclui-se que uma gestão de pessoas integrada propicia para o alinhamento entre os interesses da empresa e dos funcionários, impactando de forma positiva no desempenho da mesma.

Palavras-chave: Gestão de Pessoas. Competências Gerenciais. Agronegócio. 


\title{
COMPETENCE MANAGEMENT: A PERSPECTIVE OF MANAGERS OF AGRIBUSINESS COMPANIES
}

\begin{abstract}
The objective of this study is to identify the management competences in the perception of managers in their decision making. The theoretical framework presents a short discussion of the concepts of competences that assist in understanding the study. Data collection was performed with 149 managers of companies operating in the agribusiness sector, the technique used to analyze these data was the exploratory factor analysis, with the possible six main factors extraction that grouped the study variables differently to the initial model of the proposed study, creating a new statistical scale that can be used in future empirical research to measure the managerial skills in different contexts of study. Corroborating the literature, the study presents a framework to identify six dimensions in which managers perceive and recognize the skills needed in the process of management of agribusiness companies. Thus, it is concluded that an integrated people management provides for the alignment between the interests of the company and employees, impacting positively on its performance.
\end{abstract}

Keywords: Human Resources Management. Management Competences. Agribusiness.

\section{INTRODUÇÃO}

A competitividade nos cenários econômicos, independente do setor de atuação, mobilizou as empresas na busca por novas dinâmicas que as torne mais eficientes e eficazes dentro dos mercados sejam eles nacionais ou internacionais. Esses mesmos cenários apresentam-se para as empresas do setor de agronegócios, em que foi preciso a adoção de estratégias por aumento de produtividade sem perder o foco na qualidade e nos custos da produção (ARAÚJO, 2013; SILVA; BINOTTO, 2013; SILVA; BINOTTO; VILPOUX, 2016).

Conforme dados divulgados pelo Centro de Estudos Avançados em Economia Aplicada (CEPEA) na Universidade de São Paulo (2014), as atividades de agronegócios representam em torno de $23 \%$ do Produto Interno Bruto (PIB) brasileiro e respondem por aproximadamente $40 \%$ do faturamento das exportações brasileiras contribuindo pelos superávits comerciais do país, e a economia da grande maioria das pequenas cidades brasileiras.

O setor de agronegócios é um dos setores mais dinâmicos da economia, precisa combinar recursos naturais, tecnológicos, ambientais, econômicos, humanos, em um ambiente dependente de fatores climáticos e regulamentações que interferem diretamente na gestão das empresas em qualquer nível da cadeia produtiva. A cadeia produtiva do agronegócio é ampla e complexa, envolve os agentes que atuam nos segmentos antes, dentro e depois da porteira de forma interligada e competitiva. Devido a essa competitividade, as empresas precisam buscar a inovação para manterem-se atualizadas e assim aumentarem sua eficiência (MEGIDO; XAVIER, 2003).

Nesse sentido, se a melhoria da eficácia das empresas depende da utilização adequada de seus recursos sejam eles materiais, tecnológicos, financeiros ou humanos, a participação das pessoas nos diferentes processos da empresa torna-se fator de diferenciação, pois por meio das suas competências pode promover a busca pelo melhor desempenho.

Em um processo de gestão participativa e integrada em uma empresa que estimula a participação dos seus profissionais, as competências profissionais são as principais estratégias de gestão (BRANDÃO; GUIMARÃES, 2001), visto que podem ser entendidas como a capacidade dos indivíduos compreenderem as novas situações de trabalho de forma proativa e responsável sendo reconhecida por si e por terceiros (ZARIFIAN, 2001).

Neste processo, percebe-se que os profissionais mais envolvidos são os gestores por atuarem diretamente nas ações estratégicas das empresas, juntamente com a direção, e direcionando o trabalho junto com suas equipes, sendo assim de extrema importância o

Perspectivas em Gestão \& Conhecimento, João Pessoa, v. 7, n. 1, p. 232-247, jan./jun. 2017. 
desenvolvimento das competências individuais. Essas ações buscam adaptar as empresas frente às forças que atuam sobre o seu setor de negócios, antecipando possíveis problemas ou identificando novas oportunidades nos novos cenários que se apresentam.

Pode-se dizer que, frente a tantas competências exigidas aos profissionais gestores das empresas do agronegócio, as competências gerenciais merecem destaque, uma vez que esse gestor desempenha a função de líder da equipe. Assim, anseia-se por um gestor com um perfil que tenha a capacidade de relacionar-se de maneira interdisciplinar, agregando a técnica com a forma como os indivíduos executam e desenvolvem suas atividades (PAIVA; FERREIRA, 2013).

Visando aprofundar o entendimento das competências requeridas dos gestores que atuam diretamente na dinâmica de mercado das empresas do setor de agronegócios, o objetivo deste estudo é identificar estas competências gerenciais necessárias para estes gestores em suas tomadas de decisões a partir das suas próprias percepções. Para tanto, utilizou-se de uma pesquisa quantitativa, aplicada por meio de uma survey, com 152 gestores de empresas. Adotou-se, para a pesquisa, a Escala de Competências proposta por Quinn et al. (2003), com base nos modelos de gestão e papéis gerenciais. Os dados foram analisados por meio de análise fatorial, sendo possível explorar as variáveis do estudo.

Assim, o estudo foi estruturado a partir de seis seções. Inicia com a introdução aqui apresentada e na sequência traz o referencial teórico que aborda a teoria sobre o tema competências tendo como foco o gestor atuante em empresas do setor de agronegócios. A terceira seção expõe e descreve os procedimentos metodológicos usados na realização da pesquisa, a quarta apresenta os resultados e as análises dos dados coletados, e em seguida nas considerações finais, apresenta os principais resultados encontrados em relação ao objetivo que conduziu o estudo, assim como suas limitações e sugestões. Por fim, são apresentadas as referências que contribuíram para o embasamento do presente estudo.

\section{REFERENCIAL TEÓRICO}

Este tópico apresenta uma breve discussão dos conceitos de competências, de acordo com alguns autores que auxiliam no entendimento do contexto do estudo, focando as ideias de competências no nível gerencial, apresentando ao final o modelo de Quinn, Faerman, Thompson e McGrath (2003) usado no desenvolvimento da presente pesquisa.

\subsection{Competências}

Em busca de novos modelos de gestão surgem novas formas de gerenciamento que leva em conta as competências das lideranças em busca de construir novos caminhos e adaptá-los frente às dificuldades inesperadas (JAQUES, 1979). O tema competências surgiu dentro da área de Gestão de Pessoas e passou a ser utilizado no contexto organizacional no desenvolvimento e desempenho destas.

A origem do termo vem do latim competenia, indicando qualidade de resolver um assunto com capacidade, habilidade e aptidão (FERREIRA, 1995). A expressão competências veio a designar o reconhecimento sobre a capacidade de alguém se pronunciar a respeito de algum determinado assunto, posteriormente, também passou a ser utilizada para qualificar 0 indivíduo (BRANDÃO; GUIMARÃES, 2001).

Segundo Wood Jr. (2002), o conceito de competências surgiu designando o reconhecimento social perante a capacidade de uma pessoa em posicionar-se em relação a um assunto e assim qualificar o indivíduo como capaz para a realização de alguns trabalhos. A competência também é entendida como capacidades definidas como conhecimento, habilidades e atitudes, que desenvolvidas e mobilizadas consistem em cumprir uma demanda específica de trabalho (RUAS, 2005).

Perspectivas em Gestão \& Conhecimento, João Pessoa, v. 7, n. 1, p. 232-247, jan./jun. 2017. 
Na França, utiliza-se uma ampla definição no que tange a competência, definindo-a como um conjunto de saberes mobilizados em decorrência de uma situação de trabalho. Seus componentes são: os conhecimentos ou saberes específicos; a inteligência profissional e pessoal; os saberes colocados em prática; as aptidões; a vontade de colocar em prática e de desenvolver as competências (LUZ, 2001). Ainda definindo competências, Fleury e Fleury (2006) referem-se a um conjunto de conhecimentos (saber), habilidades (saber fazer) e atitudes (saber ser/agir) relacionados que afetam a maior parte do trabalho de uma pessoa (um papel ou responsabilidade). De acordo com esses autores, destaca-se que a competência é formada por três dimensões. As dimensões envolvem o saber, a técnica e a atitude em busca de gerar um processo de aprendizado em que as competências são fonte de valor para o indivíduo e para a organização, pois ao desenvolver as competências é possível transferir habilidade e conhecimentos a outras pessoas (SANDERBERG, 2000).

Essas dimensões estão interligadas e para que uma habilidade possa ser desenvolvida é necessário o suporte do conhecimento das técnicas e objetivos (BRANDÃO; GUIMARÃES, 2001), englobando assim o conhecimento, as habilidades e as atitudes, também chamado de CHA. Para Quinn et al. (2003, p. 24) "uma competência implica a detenção tanto de um conhecimento quanto da capacidade comportamental de agir de maneira adequada".

A competência se constitui na base da construção de um sistema de gestão de pessoas integrado em organizações que aprendem (FLEURY; FLEURY, 2004). Os autores ainda referemse a competências como o saber agir responsável e reconhecido, que implica mobilizar, integrar, transferir conhecimentos, recursos e habilidade, que agreguem valor econômico à organização e valor social ao indivíduo.

Portanto, a interação dos indivíduos com o ambiente é de extrema relevância para a obtenção de melhores resultados de desempenho. Dejours (1997) afirma que a competência humana é dependente do contexto ético e social, como também do sujeito e do coletivo, sendo assim causa de impactos significativos neste ambiente.

\subsection{Competências gerenciais no agronegócio}

O setor de agronegócios brasileiro enfrentou várias transformações e precisou inovar para adaptar-se às exigências do mercado consumidor, em especial quando se menciona a cadeia produtiva agroalimentar inserida nesse contexto. Além de inovar em tecnologias e processos, foi preciso inovar na capacitação das pessoas, principalmente de seus gestores.

O termo agronegócio passou a ser difundido no Brasil somente a partir da década de 1980 e teve sua origem pela expressão agribusiness definido por dois professores de Havard John Davis e Ray Goldberg em 1957, como um conjunto de atividades que têm início com a fabricação de insumos agropecuários, passando pela produção destes em unidades agropecuárias para que ocorra o processamento de produtos in natura em produtos industrializados e assim distribuídos até chegar ao consumidor final (ARAÚJO, 2013).

A visão sistêmica da cadeia do agronegócio mostra a necessidade de profissionais capazes de alinhar as estratégias entre os agentes envolvidos para alcançar melhores resultados frente às mudanças que incorporam uma diversidade de atividades e tecnologias (ARAÚJO, 2013). As empresas precisam especializar-se, conhecer seu ambiente e suas competências de atuação. Mas para isso, surge a necessidade de identificar as competências exigidas dos profissionais principalmente do que atuam diretamente na gestão das empresas (Amorin, 2009), as quais também podem ser identificadas como competências gerenciais.

Essas competências resultam da dimensão do conhecimento e das experiências do profissional ao desempenhar suas funções. Zarifian (2001) e Le Bortef (2003) afirmam que a competência pode ser vista a partir da capacidade do indivíduo gerar resultados percebidos pelas organizações. Também podem ser traduzidas por características pertencentes a uma

Perspectivas em Gestão \& Conhecimento, João Pessoa, v. 7, n. 1, p. 232-247, jan./jun. 2017. 
pessoa em relação ao desempenho desta em suas tarefas, diferenciando competências por: aptidões - talento natural; habilidades - talento prático; e conhecimento - talento no saber para executar uma tarefa (FLEURY; FLEURY, 2001).

O processo de formar e desenvolver competências é contínuo e implica no envolvimento de vários atores da organização (PAIVA, 2007). Assim identificar as competências requeridas pode ser entendido como mapear as competências apresentadas pelo profissional no cotidiano de seu trabalho e no contexto da sua gestão (PAIVA et al., 2014). Essas competências e habilidades tornam-se relevantes para a organização, uma vez que possibilitam conhecimento para planejar de forma estratégica a tomada de decisões, para atingir resultados esperados ao desempenho organizacional frente a mercados competitivos do contexto contemporâneo (SANTOS et al., 2016), característica atual do mercado do agronegócio.

Diante das muitas competências a serem requeridas do profissional gestor das empresas do agronegócio, as competências gerenciais são o ponto de destaque, além de gerir suas atividades na organização, desempenha a função de líder da equipe. Por isso, espera-se de um gestor um perfil capaz de interagir de maneira interdisciplinar interligando a técnica com a maneira como os indivíduos executam e desenvolvem suas funções (PAIVA; FERREIRA, 2013).

Quinn et al. (2003) apresentam um modelo de gestão falando das competências gerenciais a partir dos papéis da liderança. A síntese desse modelo pode ser entendida da seguinte forma:

1) modelo baseado em metas racionais: foca aspectos externos da organização, como produtividade, lucro, controle e o gestor exerce o papel de diretor e produtor.

2) modelo baseado em processos internos: destaca aspectos do ambiente interno da organização como integração e estabilidade organizacional, e o gestor exerce papel de monitor e coordenador.

3) modelo baseado nas relações humanas: foca aspectos internos da organização como autonomia e flexibilidade, o gestor atua como facilitador e mentor.

4) modelo baseado em sistemas abertos: onde a organização focaliza aspectos de diferenciação e flexibilidade do seu ambiente externo, o gestor atua como negociador e inovador com poder de influência.

Neste contexto, a apresentação destes modelos por meio de oito papéis gerenciais a que compete a atividade do gestor, conforme pode ser observado no Quadro 1.

Quadro 1 - Modelos de gestão e papéis gerenciais

\begin{tabular}{|c|ll|}
\hline Papéis & \multicolumn{2}{|c|}{ Competências } \\
\hline \multirow{3}{*}{ Papel de mentor } & 1. & Compreensão de si mesmo e dos outros \\
\cline { 2 - 3 } & 2. & Comunicação eficaz \\
\cline { 2 - 3 } & 3. & Desenvolvimento dos empregados \\
\hline \multirow{2}{*}{ Papel de facilitador } & 1. & Construção de equipes \\
\cline { 2 - 3 } & 2. & Uso do processo decisório participativo \\
\cline { 2 - 3 } & 3. & Administração de conflitos \\
\hline \multirow{2}{*}{ Papel de monitor } & 1. & Monitoramento do desempenho individual \\
\cline { 2 - 3 } & 2. & Gerenciamento do desempenho e processos coletivos \\
\hline \multirow{2}{*}{ Papel de coordenador } & 1. & Gerenciamento de projetos \\
\cline { 2 - 3 } & 2. & Planejamento do trabalho \\
\cline { 2 - 3 } & 3. & Gerenciamento multidisciplinar \\
\hline \multirow{2}{*}{ Papel de diretor } & 1. & Desenvolvimento e comunicação de uma visão \\
\cline { 2 - 3 } & 2. & Estabelecimento de metas e objetivos \\
\hline
\end{tabular}

Perspectivas em Gestão \& Conhecimento, João Pessoa, v. 7, n. 1, p. 232-247, jan./jun. 2017. 


\begin{tabular}{|c|ll|}
\hline \multirow{3}{*}{ Papel de produtor } & 3. & Planejamento e organização \\
\cline { 2 - 3 } & 1. & Trabalho produtivo \\
\cline { 2 - 3 } & 2. & Fomento de um ambiente de trabalho produtivo \\
\cline { 2 - 3 } Papel de negociador & 3. & Gerenciamento de tempo e do estresse \\
\cline { 2 - 3 } & 1. & Construção e manutenção de uma base de poder \\
\cline { 2 - 3 } & 2. & Negociação de acordos e compromissos \\
\cline { 2 - 3 } & 3. & Apresentação de ideais \\
\hline \multirow{2}{*}{ Papel de inovador } & 1. & Convívio com a mudança \\
\cline { 2 - 3 } & 2. & Pensamento criativo \\
\cline { 2 - 3 } & 3. & Gerenciamento da mudança \\
\hline
\end{tabular}

Fonte: Quinn et al. (2003, p. 25)

Em cada um dos papéis espera-se o desenvolvimento de três competências gerenciais que contribuem para o desenvolvimento dos gestores e assim de suas competências, atendendo de forma contínua as demandas específicas do cotidiano destes profissionais (QUINN et al., 2003), caracterizando as competências gerencias aqui estudadas.

\section{PROCEDIMENTOS METODOLÓGICOS}

A realização de um estudo científico constitui-se fundamentalmente pelo método aplicado, onde são relacionados os procedimentos adotados para atender os objetivos propostos no estudo e a fundamentação teórica que embasa os conceitos abordados. A presente pesquisa caracteriza-se pela utilização de uma abordagem quantitativa, de natureza exploratória em que foi possível identificar correlações entre o fenômeno estudado, competências gerenciais, e também fornecer novas contribuições para o mesmo (ROESCH, 1999; RICHARDSON, 1999). Neste cenário, também, caracteriza-se pelo estudo descritivo, pois permitiu descrever, analisar e interpretar o tema competências a partir do contexto de agronegócios ampliando os conhecimentos já existentes e estabelecendo relações entre as variáveis estudadas (TRIVIÑOS, 1987; MARCONI; LAKATOS, 2003).

Visando identificar as competências mencionadas na percepção dos gestores das empresas do agronegócio, foi realizado um levantamento bibliográfico sobre o tema, que permitiu a identificação do construto (Quadro 2) da presente pesquisa a partir de 19 variáveis enquadradas conforme categorização proposta pelo modelo de QUINN et al., (2003) adaptadas a este estudo com o intuito de medir de forma quantitativa na população do contexto estudado.

Como população desta pesquisa, foram selecionados profissionais de empresas do agronegócio atuantes em diferentes atividades deste setor na região norte do Rio Grande do Sul. Trata-se de uma amostra não probabilística por conveniência (MALHOTRA, 2006), considerando apenas a característica de serem profissionais que atuam como gestores destas empresas.

Quadro 2 - Variáveis da pesquisa

\begin{tabular}{|c|l|}
\hline \multicolumn{1}{|c|}{ CONSTRUTOS } & \multicolumn{1}{|c|}{ VARIÁVEIS } \\
\hline \multirow{4}{*}{ PAPEL DE MENTOR } & $\begin{array}{l}\text { Q1-Em meus relacionamentos busco desenvolver a empatia com meus } \\
\text { superiores, subordinados, clientes e demais pares de trabalho. }\end{array}$ \\
\cline { 2 - 2 } & Q2-Mantenho uma comunicação clara e objetiva. \\
\cline { 2 - 2 } & $\begin{array}{l}\text { Q3-Contribuo com as pessoas para aumentar a sua consciência sobre suas } \\
\text { potencialidades e necessidades de desenvolvimento pessoal e profissional. }\end{array}$ \\
\hline \multirow{2}{*}{ Papel de Facilitador } & $\begin{array}{l}\text { Q4-Exerço influência como líder nos argumentos apresentados pela equipe de } \\
\text { trabalho. }\end{array}$ \\
\hline
\end{tabular}

Perspectivas em Gestão \& Conhecimento, João Pessoa, v. 7, n. 1, p. 232-247, jan./jun. 2017. 


\begin{tabular}{|c|c|}
\hline & Q5-Encontro soluções novas frente aos problemas. \\
\hline \multirow[t]{2}{*}{ Papel de Monitor } & $\begin{array}{l}\text { Q6-Reconheço os esforços de meus funcionários enfatizando o acerto na busca } \\
\text { da correção do erro. }\end{array}$ \\
\hline & Q7-Gerencio o desempenho dos processos da minha equipe de trabalho. \\
\hline \multirow{2}{*}{$\begin{array}{l}\text { Papel de } \\
\text { Coordenador }\end{array}$} & Q8-Gerencio projetos de forma dinâmica. \\
\hline & Q9-Adapto o planejamento do trabalho conforme atividades necessárias. \\
\hline \multirow[t]{3}{*}{ Papel de Diretor } & $\begin{array}{l}\text { Q10-Desenvolvo e comunico de forma clara minha visão aos públicos da } \\
\text { organização. }\end{array}$ \\
\hline & Q11-Estabeleço metas e objetivos de trabalho. \\
\hline & Q12-Planejo e organizo minhas atividades. \\
\hline \multirow[b]{2}{*}{ Papel de Produtor } & Q13-Fomento um ambiente de trabalho produtivo. \\
\hline & $\begin{array}{l}\text { Q14-Programo prioridades para cumprir prazos e objetivos sem gerar um } \\
\text { ambiente de estresse. }\end{array}$ \\
\hline \multirow{3}{*}{$\begin{array}{l}\text { Papel de } \\
\text { Negociador }\end{array}$} & Q15-Consigo influenciar a partir da apresentação das minhas ideias. \\
\hline & Q16-Estabeleço compromissos sustentáveis e negociações eficazes. \\
\hline & Q17-Construo minhas relações de poder a partir de relações participativas. \\
\hline \multirow[t]{2}{*}{ Papel de Inovador } & $\begin{array}{l}\text { Q18-Considero-me um profissional criativo na busca por novos desafios e } \\
\text { soluções. }\end{array}$ \\
\hline & $\begin{array}{l}\text { Q19-Trabalho facilmente com mudanças que ocorrem no ambiente interno e } \\
\text { externo. }\end{array}$ \\
\hline
\end{tabular}

Fonte: Adaptado de Quinn et al. (2003)

Quanto aos procedimentos de coleta de dados, utilizou-se de uma survey (FREITAS, et al., 2000) onde os dados foram coletados por meio da percepção dos gestores pesquisados. 0 instrumento utilizado para aplicação da survey, foi um questionário elaborado a partir do construto do estudo, contendo 19 questões fechadas (numeradas de Q1 à Q19), onde foi adotada uma escala tipo likert expandida em 6 pontos. A escala de 6 pontos mede os valores entre 1 a 6 , sendo que 1 corresponde a discordo totalmente e 5 concordo totalmente, a pontuação 6 indica a resposta sem condições de opinar.

Para uma melhor aplicação do questionário, foi realizado um pré-teste (FREITAS, et al., 2000) com 3 gestores, no intuito de verificar se o mesmo apresentava uma linguagem adequada, clara e de fácil resposta para alcançar o objetivo proposto. Esta aplicação foi positiva, não havendo alterações no mesmo. A forma de aplicação da pesquisa foi presencial, onde os questionários foram entregues aos gestores para posteriormente serem respondidos. Após o período de uma semana, os mesmos foram recolhidos, e dos 250 questionários entregues, obteve-se um retorno de 152 questionários (60,8\%), onde destes 149 foram validados para compor a amostra. A aplicação dos questionários para a coleta dos dados, aconteceu entre os meses de maio a junho de 2015.

Para tabulação dos dados obtidos foi utilizado uma planilha Excel e posteriormente analisados por meio do software SPSS - Statistical Package for the Social Sciences. Inicialmente, foram analisadas as estatísticas descritivas das variáveis verificando eventuais erros de digitação, como também estatísticas descritivas da caracterização dos respondentes da pesquisa. Não identificado nenhum valor fora da normalidade da escala, procedeu-se com a verificação da concordância dos respondentes realizando a média e o desvio padrão das variáveis. Posteriormente foram examinados alguns critérios necessários para realização da análise fatorial, técnica de análise escolhida para este estudo, que se caracteriza como medidas estatísticas que exploram a relação entre as variáveis do estudo e podem compor um novo conjunto de fatores (HAIR Jr. et al., 1998). 
O primeiro critério avaliado foi o tamanho da amostra, para cada variável analisada o critério é que tenha pelo menos 5 respondentes (TABACHNICK; FIDELL, 1989; PASQUALI, 2005), sendo que na presente pesquisa atingiu-se 7,84 respondentes para cada variável, atendendo assim a esse requisito. Em seguida, explorou-se o grau de confiabilidade de Alpha Cronbach (PETERSON, 1994) coeficiente que mede a consistência das variáveis do estudo onde aponta se $\mathrm{o}$ instrumento de medida produz resultados coerentes. Também realizou-se os testes Kaiser-Meyer-Olkin (KMO) e de Esfericidade de Bartlett (HUTCHESON; SORONIOU, 1999; HAIR et al., 2005) confirmando a análise fatorial varimax apropriada como técnica de análise a partir dos valores obtidos. Contudo, a partir da análise fatorial varimax pode-se observar a comunalidade (LEE; HOOLEY, 2005) dos dados e a carga fatorial de cada variável, compondo cada fator (HAIR Jr. et al., 1998), justificando assim a escolha da técnica de análise fatorial com rotação varimax para a presente pesquisa.

\section{ANÁLISE E DISCUSSÃO DOS RESULTADOS}

Para a análise desta pesquisa utilizou-se da técnica de análise fatorial exploratória que permitiu verificar a percepção entre os gestores do setor do agronegócio acerca das competências gerenciais requeridas e medidas por meio do instrumento apresentado. De acordo com a Tabela 1, do total de 149 gestores respondentes das empresas do agronegócio são predominantemente do gênero masculino, sendo que estes representam 123 homens $(82,6 \%)$ e apenas 26 mulheres $(17,4 \%)$. Em relação ao grau de escolaridade, os gestores apresentam maior número de formação em ensino médio completo 58 gestores (38,9\%), ensino superior completo 42 gestores $(28,2 \%)$, pós-graduação latu senso 25 gestores $(16,8 \%)$, e 24 gestores $(16,1 \%)$ possuem escolaridade abaixo do ensino médio completo. Dentre estes respondentes, percebe-se que 65 deles (46,3\%) atuam no cargo gerencial entre 6 a 20 anos o que demonstra uma maturidade profissional dos mesmos, requerida nas competências gerenciais estudadas.

Analisando as características das empresas em que estes gestores atuam, nota-se que as atividades destas se concentram no atendimento direto a propriedade rural em atividades como: comércio de insumos $(31,5 \%)$, indústria e comércio de máquinas e implementos agrícolas $(24,8 \%)$, cooperativas $(12,8 \%)$ e cerealistas (beneficiamento e comércio de cereais $11,4 \%)$. O quadro de pessoal destas empresas é composto por: até 10 funcionários $(34,2 \%)$ de 11 a 100 funcionários (32,9\%), de 101 a 500 funcionários (14,8\%), de 501 a 1000 funcionários $(6,7 \%)$ e acima de 1000 funcionários $(11,4 \%)$.

Tabela 1 - Caracterização da amostra

\begin{tabular}{|c|c|c|c|}
\hline \multicolumn{4}{|c|}{ Gênero } \\
\hline & & requência & Percentual \\
\hline Masculino & & 123 & $82,6 \%$ \\
\hline Feminino & & 26 & $17,4 \%$ \\
\hline \multicolumn{4}{|c|}{ Escolaridade } \\
\hline & & requência & Percentual \\
\hline Ensino médio completo & & 58 & $38,9 \%$ \\
\hline Superior completo & & 42 & $28,2 \%$ \\
\hline Pós-Graduação lato sensu & & 25 & $16,8 \%$ \\
\hline Abaixo de ensino médio & & 24 & $16,1 \%$ \\
\hline \multicolumn{4}{|c|}{ Atividade das empresas e número de funcionários } \\
\hline Atividade & Percentual & Nr. De Funcionários & Percentual \\
\hline Comércio de insumos agrícolas & $31,5 \%$ & Até 10 & $34,2 \%$ \\
\hline
\end{tabular}

Perspectivas em Gestão \& Conhecimento, João Pessoa, v. 7, n. 1, p. 232-247, jan./jun. 2017. 
Lidiane Cássia Comin et al.

\begin{tabular}{|c|c|c|c|}
\hline Ind. e com. de máquinas e implementos agrícolas & $24,8 \%$ & De 11 à 100 & $32,9 \%$ \\
\hline Cooperativas & $12,8 \%$ & De 101 à 500 & $14,8 \%$ \\
\hline Cerealistas & $11,4 \%$ & De 501 à 1.000 & $6,7 \%$ \\
\hline Outras & $19,5 \%$ & Acima de 1.000 & $11,4 \%$ \\
\hline
\end{tabular}

Fonte: Dados da pesquisa (2015)

Após a análise dos dados e a constatação da normalidade dos mesmos sem que houvesse a necessidade de tratamento destes, extraiu-se a média e o desvio padrão das variáveis. Conforme nota-se na Tabela 2 , há uma alta concordância entre os respondentes em relação aos questionamentos feitos comprovando-se por meio dos valores do desvio padrão das mesmas em menor que 1.

Tabela 2 - Média e desvio padrão das variáveis do estudo

\begin{tabular}{c|c|c|c}
\hline & Média & Desvio Padrão & Análise N \\
\hline Q1 & 4,41 & 0,878 & 149 \\
\hline Q2 & 4,66 & 0,579 & 149 \\
\hline Q3 & 4,58 & 0,595 & 149 \\
\hline Q4 & 4,56 & 0,574 & 149 \\
\hline Q5 & 4,42 & 0,649 & 149 \\
\hline Q6 & 4,63 & 0,562 & 149 \\
\hline Q7 & 4,53 & 0,643 & 149 \\
\hline Q8 & 4,29 & 0,671 & 149 \\
\hline Q9 & 4,59 & 0,668 & 149 \\
\hline Q10 & 4,48 & 0,674 & 149 \\
\hline Q11 & 4,46 & 0,692 & 149 \\
\hline Q12 & 4,52 & 0,643 & 149 \\
\hline Q13 & 4,64 & 0,535 & 149 \\
\hline Q14 & 4,30 & 0,723 & 149 \\
\hline Q15 & 4,36 & 0,658 & 149 \\
\hline Q16 & 4,51 & 0,759 & 149 \\
\hline Q17 & 4,36 & 0,763 & 149 \\
\hline Q18 & 4,52 & 0,588 & 149 \\
\hline Q19 & 4,38 & 0,702 & \\
\hline
\end{tabular}

Fonte: Dados da pesquisa extraídos via relatório do SPSS (2015)

Para o desenvolvimento da análise fatorial exploratória foram utilizadas 19 variáveis conforme apresentadas no Quadro 1 . Na validação das variáveis verificou-se a consistência destas através do Alpha de Cronbach, que mede a consistência entre as múltiplas medidas de uma variável, tendo como aceitável o valor de 0,7 (HAIR et al., 2005). Porém, em alguns cenários das ciências sociais considera-se esse valor a partir de 0,6 (PETERSON, 1994) sendo que nesta pesquisa o índice encontrado foi de 0,836 (Tabela 3), considerado como ideal e comprovando assim sua confiabilidade.

Outro critério apresentado na Tabela 3 são os testes de KMO e Bartlett, onde apresentam índices satisfatórios para a realização da análise fatorial. O índice KMO apresenta o valor de 0,776, considerado como um bom índice e adequado para a técnica de análise fatorial segundo Hutcheson e Soroniou (1999). Para estes autores, interpretam os índices de KMO, dizendo que valores menores que 0,5 não são considerados adequados para a técnica de análise fatorial, valores entre 0,5 e 0,7 são considerados baixos; valores entre 0,7 e 0,8 são considerados bons; valores maiores que 0,8 e 0,9 são considerados ótimos. Quanto ao Teste

Perspectivas em Gestão \& Conhecimento, João Pessoa, v. 7, n. 1, p. 232-247, jan./jun. 2017. 
de Esfericidade de Bartlett o grau de significância encontrado foi de 0,000, o que segundo Hair et al. (2005) esse valor abaixo de 0,05 indica adequação do uso da análise fatorial.

Tabela 3 - Resultado do teste Alpha de Cronbach, KMO, Esfericidade de Bartlett e percentual de variância explicada das variáveis do estudo.

\begin{tabular}{c|c|c|c|c}
\hline Alpha de Cronbach & \multirow{2}{*}{ KMO } & \multicolumn{2}{|c|}{ Esfericidade de Bartlett } & \multirow{2}{*}{ \% Var. Explicada } \\
\cline { 3 - 4 } & Qui quadrado & Sig. & \\
\hline 0,836 & 0,776 & 750,511 & 0,000 & $60,612 \%$ \\
\hline
\end{tabular}

Fonte: Dados da pesquisa extraídos via relatório do SPSS (2015)

O percentual de variância explicada das variáveis demonstrado na Tabela 3, tem o objetivo de explicar o fator em relação ao montante específico de variância em relação as variáveis relacionadas a este, considerando que a partir de $60 \%$ é satisfatório esse percentual (Hair et al., 2005). A partir da Rotação Varimax livre realizada com os dados da presente pesquisa, foi possível a extração de seis fatores principais, no qual cada variável apresenta uma carga fatorial entre 0,502 e 0,804, acima de 0,4 que segundo Hair Jr. et al. (1998) é considerado um valor aceitável para a realização desta técnica de análise, sendo que o coeficiente da carga fatorial mede a correlação entre as variáveis e os respectivos fatores a que estas foram agrupadas. Assim, conforme Tabela 4, apresenta-se estes fatores com 0 percentual de variância e a carga fatorial extraída livremente para cada variável nesta pesquisa.

Tabela 4 - Fatores extraídos na pesquisa com a carga fatorial de cada variável

\begin{tabular}{|c|c|c|c|c|c|}
\hline Fator (novo construto) & Total & \% variância & $\%$ cumulativa & \multicolumn{2}{|c|}{ Carga fatorial de cada variáve } \\
\hline \multirow{4}{*}{ Fator 1} & \multirow{4}{*}{5,015} & \multirow{4}{*}{26,397} & \multirow{4}{*}{26,397} & Q9 & 0,797 \\
\hline & & & & Q11 & 0,510 \\
\hline & & & & Q12 & 0,688 \\
\hline & & & & Q14 & 0,502 \\
\hline \multirow[b]{2}{*}{ Fator 2} & \multirow[b]{2}{*}{1,509} & \multirow[b]{2}{*}{7,943} & \multirow[b]{2}{*}{34,340} & Q18 & 0,684 \\
\hline & & & & Q19 & 0,782 \\
\hline \multirow{5}{*}{ Fator 3} & \multirow{5}{*}{1,425} & \multirow{5}{*}{7,499} & \multirow{5}{*}{41,839} & Q7 & 0,526 \\
\hline & & & & Q8 & 0,558 \\
\hline & & & & Q10 & 0,684 \\
\hline & & & & Q16 & 0,670 \\
\hline & & & & Q17 & 0,581 \\
\hline \multirow[b]{2}{*}{ Fator 4} & \multirow[b]{2}{*}{1,306} & \multirow[b]{2}{*}{6,873} & \multirow[b]{2}{*}{48,712} & Q5 & 0,629 \\
\hline & & & & Q6 & 0,804 \\
\hline \multirow{3}{*}{ Fator 5} & \multirow{3}{*}{1,183} & \multirow{3}{*}{6,226} & \multirow{3}{*}{54,937} & Q3 & 0,578 \\
\hline & & & & Q4 & 0,692 \\
\hline & & & & Q15 & 0,713 \\
\hline \multirow{3}{*}{ Fator 6} & \multirow{3}{*}{1,078} & \multirow{3}{*}{5,674} & \multirow{3}{*}{60,612} & Q1 & 0,787 \\
\hline & & & & Q2 & 0,604 \\
\hline & & & & Q13 & 0,445 \\
\hline
\end{tabular}

Fonte: Dados da pesquisa extraídos via relatório do SPSS (2015)

Em relação aos valores extraídos da comunalidade das variáveis, os mesmos apresentam valores superiores a média, apenas a Q5 apresentou um valor inferior à 0,5 de 0,441 . A comunalidade representa a variância total de uma variável compartilhada com as demais que compõem o instrumento sendo os valores aceitáveis entre 0 e 1 , quanto mais próximos de 1 mais indicam que todas as variâncias são explicadas pelos fatores comuns (HAIR 
et al., 1998). A Tabela 5 apresenta os valores da comunalidade extraídos das variáveis a partir dos seis componentes apontados.

Tabela 5 - Comunalidade das variáveis de estudo

\begin{tabular}{c|c|c}
\hline Variável & Inicial & Extração \\
\hline Q1 & 1,00 & 0,650 \\
\hline Q2 & 1,00 & 0,542 \\
\hline Q3 & 1,00 & 0,537 \\
\hline Q4 & 1,00 & 0,659 \\
\hline Q5 & 1,00 & 0,441 \\
\hline Q6 & 1,00 & 0,734 \\
\hline Q7 & 1,00 & 0,604 \\
\hline Q8 & 1,00 & 0,580 \\
\hline Q9 & 1,00 & 0,708 \\
\hline Q10 & 1,00 & 0,593 \\
\hline Q11 & 1,00 & 0,516 \\
\hline Q12 & 1,00 & 0,671 \\
\hline Q13 & 1,00 & 0,532 \\
\hline Q14 & 1,00 & 0,546 \\
\hline Q15 & 1,00 & 0,648 \\
\hline Q16 & 1,00 & 0,598 \\
\hline Q17 & 1,00 & 0,667 \\
\hline Q18 & 1,00 & 0,590 \\
\hline Q19 & 1,00 & 0,702 \\
\hline
\end{tabular}

Fonte: Dados da pesquisa extraídos via relatório do SPSS (2015)

A partir deste contexto, foi possível também a análise entre as variáveis que compõem cada fator, explicando as correlações existentes em cada agrupamento, apresentando-se os valores dos testes e das cargas fatoriais das variáveis dentro do grupo. Destes seis fatores extraídos, os mesmos foram identificados a partir de uma dimensão que expressa características relacionadas ao conhecimento, a habilidade e a atitude dos gestores no desenvolvimento de suas competências agrupando as variáveis para cada um deles conforme a Tabela 6.

Tabela 6 - Análise fatorial - Rotação Varimax

\begin{tabular}{|c|c|c|c|c|c|}
\hline Fator 1 & Variáveis & Média & Desvio Padrão & Comunalidades & Carga Fatorial \\
\hline \multirow{4}{*}{ Dimensão Hierárquica } & Q9 & 4,59 & 0,668 & 0,387 & 0,622 \\
\hline & Q11 & 4,46 & 0,692 & 0,565 & 0,752 \\
\hline & Q12 & 4,52 & 0,643 & 0,656 & 0,810 \\
\hline & Q14 & 4,30 & 0,723 & 0,581 & 0,762 \\
\hline \multicolumn{6}{|c|}{ Alfa Cronbach: 0,719 } \\
\hline Fator 2 & Variáveis & Média & Desvio Padrão & Comunalidades & Carga Fatorial \\
\hline \multirow{2}{*}{ Dimensão Inovativa } & Q18 & 4,52 & 0,588 & 0,718 & 0,848 \\
\hline & Q19 & 4,38 & 0,702 & 0,718 & 0,848 \\
\hline \multicolumn{2}{|c|}{ Alfa Cronbach: 0,601 } & \multicolumn{4}{|c|}{ Nível de significância: $\mathbf{0 , 0 0 0}$} \\
\hline Fator 3 & Variáveis & Média & Desvio Padrão & Comunalidades & Carga Fatorial \\
\hline \multirow{4}{*}{ Dimensão Comportamental } & Q7 & 4,53 & 0,643 & 0,538 & 0,733 \\
\hline & Q8 & 4,29 & 0,671 & 0,532 & 0,730 \\
\hline & Q10 & 4,48 & 0,674 & 0,320 & 0,565 \\
\hline & Q16 & 4,51 & 0,759 & 0,586 & 0,766 \\
\hline
\end{tabular}

Perspectivas em Gestão \& Conhecimento, João Pessoa, v. 7, n. 1, p. 232-247, jan./jun. 2017. 


\begin{tabular}{|c|c|c|c|c|c|}
\hline & Q17 & 4,36 & 0,763 & 0,403 & 0,635 \\
\hline \multicolumn{2}{|c|}{ Alfa Cronbach: 0,719 } & \multicolumn{3}{|c|}{ Nível de significância: 0,0} & 00 \\
\hline Fator 4 & Variáveis & Média & Desvio Padrão & Comunalidades & Carga Fatorial \\
\hline \multirow{2}{*}{ Dimensão Gerencial } & Q5 & 4,42 & 0,649 & 0,660 & 0,812 \\
\hline & Q6 & 4,63 & 0,562 & 0,660 & 0,812 \\
\hline \multicolumn{2}{|c|}{ Alfa Cronbach: 0,481 } & \multicolumn{2}{|c|}{ KMO: 0,500 } & significância: 0,00 & \\
\hline Fator 5 & Variáveis & Média & Desvio Padrão & Comunalidades & Carga Fatorial \\
\hline \multirow{3}{*}{ Dimensão Dinâmica } & Q3 & 4,58 & 0,595 & 0,586 & 0,765 \\
\hline & Q4 & 4,56 & 0,574 & 0,595 & 0,771 \\
\hline & Q15 & 4,36 & 0,658 & 0,371 & 0,609 \\
\hline \multicolumn{2}{|c|}{ Alfa Cronbach: 0,521 } & \multicolumn{2}{|c|}{ KMO: 0,590 } & significância: 0,00 & \\
\hline Fator 6 & Variáveis & Média & Desvio Padrão & Comunalidades & Carga Fatorial \\
\hline \multirow{3}{*}{ Dimensão Comunicativa } & Q1 & 4,41 & 0,878 & 0,470 & 0,686 \\
\hline & Q2 & 4,66 & 0,579 & 0,582 & 0,763 \\
\hline & Q13 & 4,64 & 0,535 & 0,475 & 0,690 \\
\hline
\end{tabular}

Fonte: Dados da pesquisa extraídos via relatório do SPSS (2015)

Analisando o conteúdo das variáveis que compõem cada fator principal é possível observar que o Fator 1, identificado aqui como dimensão hierárquica, descreve as competências requeridas à um gestor, relacionadas ao planejamento do trabalho em busca do atingimento das metas e o alinhamento entre os interesses estratégicos da empresa para uma melhor performance expressa por meio dos resultados alcançados, corroborando com as afirmações de Quinn et al. (2013) ao descrever as competências do líder apresentadas pelos papéis gerenciais de diretor e produtor. Estas variáveis possuem cargas fatoriais acima de 0,6 0 que explica suas relações com o fator e ainda representam $26,397 \%$ da variância total composto por 4 variáveis.

O Fator 2 possui dois itens, porém apresenta uma carga fatorial acima de 0,8 o que demonstra uma excelente relação entre suas variáveis. Identificado como dimensão inovativa, tais variáveis expressam a pró-atividade e a flexibilidade do gestor frente a um contexto de constante mudanças que apresenta-se nas empresas atualmente, principalmente ao setor de agronegócios devido a complexidade da sua cadeia produtiva (ARAÚJO, 2013), e as mudanças econômicas e políticas que geram influências direta, demandando inovar constantemente para que as empresas possam manterem-se atualizadas e assim aumentarem sua eficácia no mercado (MEGIDO; XAVIER, 2003).

Já o Fator 3, com 7,499\% de representação da variância total, constituído por 5 variáveis, relacionadas positivamente com o fator, apresenta uma carga fatorial entre 0,565 e 0,766 . Denominado aqui de dimensão comportamental, este fator representa as competências do gestor nos relacionamentos com sua equipe de trabalho, proporcionado um ambiente participativo e dinâmico fortalecendo as relações entre a empresa e seus funcionários, contribuindo para o alinhamento dos interesses comuns entre ambos, proporcionando uma gestão de pessoas integrada (FLEURY; FLEURY, 2004).

Os Fatores 4 e 5 identificados como dimensão gerencial e dimensão dinâmica respectivamente, apresentam uma carga fatorial acima de 0,8 , tendo 2 e 3 variáveis. Juntos explicam 13,099\% da variância total e indicam as competências requeridas aos gestores no desenvolvimento das suas lideranças, contribuindo com o desenvolvimento pessoal e 
profissional da sua equipe e ainda exercendo sua persuasão para o envolvimento de todos em busca dos objetivos propostos. Esta dimensão contribui de modo significativo com as definições apresentadas por Quinn et al. (2003), ao dizer que o gestor atua como negociador e inovador com poder de influência sobre o ambiente que atua.

O último fator extraído pela rotação, foi o Fator 6 , chamado dimensão comunicativa. Composto por 3 itens apresenta carga fatorial entre suas variáveis de 0,686 à 0,763 e explicase por $5,674 \%$ da variância total, acumulando $60,612 \%$ de explicação das variáveis por estes fatores extraídos. As competências relacionadas a este fator buscam fomentar a produtividade por meio de uma relação transparente e objetiva, com uma comunicação aberta visando a busca pela excelência do trabalho com os diferentes públicos da empresa. Assim, é possível afirmar que as competências de um gestor não limitam-se ao conhecimento adquirido, sendo necessário saber fazer e ainda desenvolver ações que promovam este saber, interdisciplinaridade entre técnica e atitude (FLEURY; FLEURY, 2006; PAIVA; FERREIRA, 2013), atuando como agente de mudança do ambiente organizacional que atua (ZUCARRI; BELLUZZO, 2016).

Diante da apresentação deste cenário, é possível a identificação de um framework que apresenta um modelo de competências gerenciais, gerado a partir da extração dos seis fatores que explicam essas competências por meio das dimensões aqui apresentadas, conforme o conjunto de variáveis pertencentes a cada uma.

Figura 1 - As dimensões das competências gerenciais

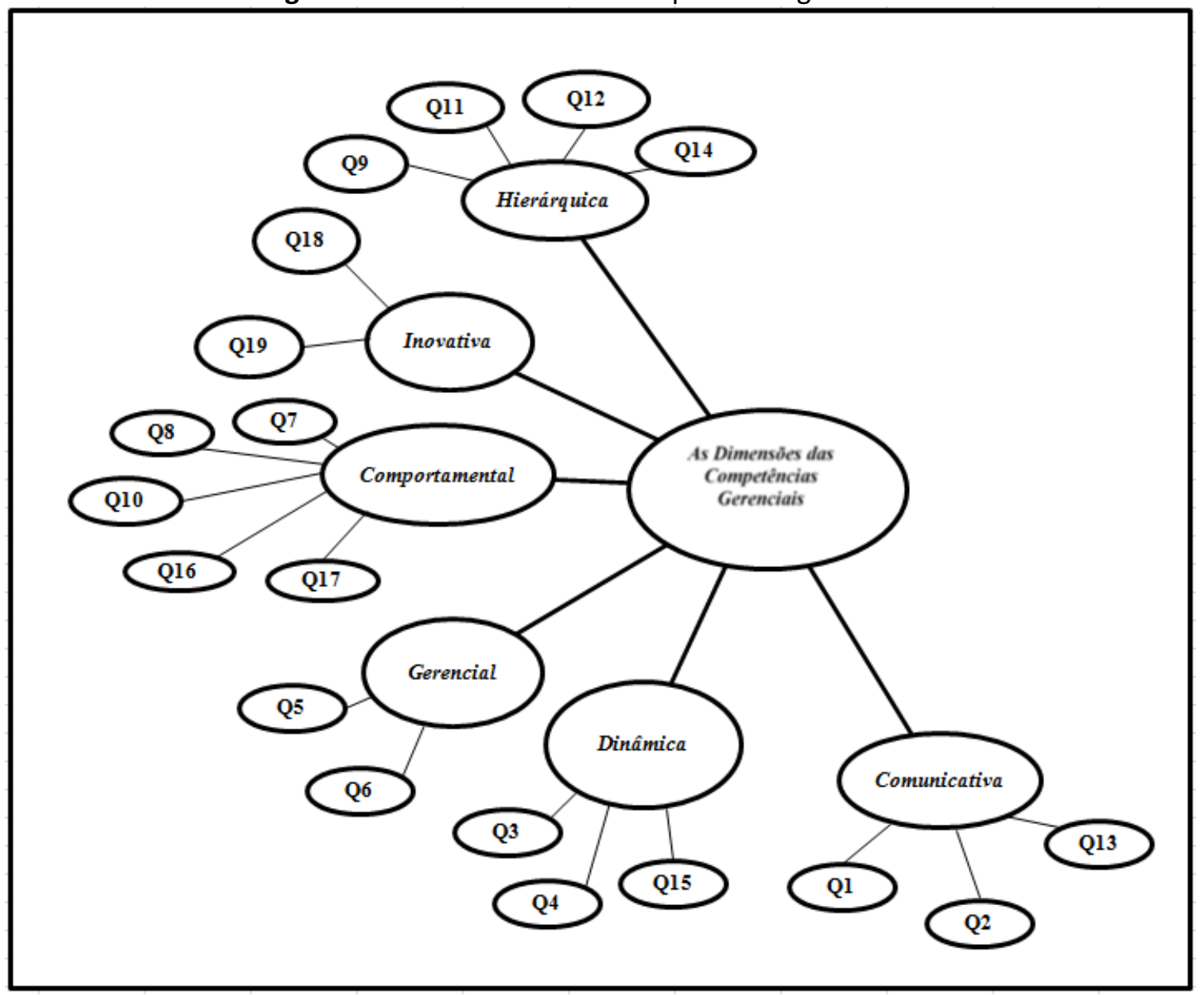

Fonte: Elaborado pelos autores a partir dos dados da pesquisa (2015)

Perspectivas em Gestão \& Conhecimento, João Pessoa, v. 7, n. 1, p. 232-247, jan./jun. 2017. 


\section{CONSIDERAÇÕES FINAIS}

A partir do objetivo proposto neste estudo, de identificar as competências gerenciais requeridas dos gestores do setor do agronegócio, sob suas próprias perspectivas, é possível concluir que estes gestores percebem e reconhecem a importância do conhecimento nos processos, no mercado e no desenvolvimento das suas equipes, liderando e influenciando de forma ativa o processo de gestão. No contexto organizacional em que a mudança é uma constante, o processo de gerenciar exige atualização permanente dos profissionais. A gestão de pessoas propicia para o alinhamento estratégico dos interesses entre empresas e funcionários, o que influencia de forma positiva no desempenho da organização. Portanto, espera-se que este estudo seja fonte de informações gerenciais para gestores e profissionais que buscam o desenvolvimento profissional na trajetória das suas carreiras, bem como para as empresas que estão em busca de novas formas de gestão encorajando seus funcionários no desenvolvimento das suas competências.

Pela análise realizada dos dados da pesquisa, utilizando-se da técnica de análise fatorial exploratória, foi possível a extração de seis fatores principais que agruparam as variáveis do estudo de forma diferenciada ao modelo inicial do estudo proposto por Quinn et al. (2003), criando assim uma nova escala estatística podendo ser utilizada em outras investigações empíricas para mensurar as competências gerencias aplicadas a diferentes contextos de estudo. Também como contribuição acadêmica identificou-se um novo framework das competências gerenciais apresentados aqui por seis dimensões geradas e confirmadas pela análise estatística adotada. Assim, este modelo corrobora com a literatura apresentada no estudo de Quinn et al. (2003), como também com os demais autores mencionados no referencial deste estudo que já abordam de forma abrangente este conceito.

Devido à relevância do tema abordado para as empresas que inovam em seus processos de gestão, as limitações deste estudo se caracterizaram em dois fatores, primeiramente quanto a obtenção de uma amostra superior, pelo fato da coleta dos dados ter sido presencial, os autores esperavam um maior número de respondente. Outra limitação apresentada neste estudo é que representa um setor específico da economia, por isso, transformado em uma sugestão para pesquisas futuras, sugere-se que esta pesquisa seja fonte de novas pesquisas envolvendo a percepção de outros profissionais que atuam em cargos de gestão, utilizando-se do instrumento criado por este estudo, aprimorando assim o conhecimento na área de competências gerenciais.

\section{REFERÊNCIAS}

ARAÚJO, M. J. Fundamentos de agronegócios. 4. ed. São Paulo: Atlas, 2013.

AMORIM, T. N. G. F. Gestão de pessoas no agronegócio. In: Callado, Antônio André Cunha (Org.). Agronegócio. 2. ed. São Paulo: Atlas, 2009.

BRANDÃO, H. P.; GUIMARÃES, T. de A. Gestão de competências e gestão de desempenho: tecnologias distintas ou instrumentos de um mesmo construto. Revista de Administração de Empresas, v. 41, n. 1, p. 9, 2001.

FLEURY, M. T. L.; FLEURY, A. C. C. Construindo o conceito de competência. Revista de administração contemporânea, v. 5, n. SPE, p. 183-196, 2001.

FLEURY, M. T. L.; FLEURY, A. C. C. Alinhando estratégia e competências. Revista de Administração de Empresas, v. 44, n. 1, p. 44-57, 2004.

Perspectivas em Gestão \& Conhecimento, João Pessoa, v. 7, n. 1, p. 232-247, jan./jun. 2017. 
FLEURY, A. C. C.; FLEURY, M. T. L. Estratégias empresariais e formação de competências. 3. ed. São Paulo: Atlas, 2006.

FERREIRA, A. B. de H. Novo dicionário básico da língua portuguesa. Rio de Janeiro: Nova Fronteira, 1995.

FREITAS, H.; OLIVEIRA, M.; SACCOL, A. Z.; MOSCAROLA, J. O método de pesquisa survey. Revista de Administração, Universidade de São Paulo, v. 35, n. 3, p. 105-112, 2000.

HAIR Jr., J. F.; ANDERSON, R. E.; TATHAM, R. L.; BLACK, W. C. Multivariate data analysis with readings. Englewood Cliffs, Prentice Hall, New Jersey, 1995.

HAIR Jr., J. F.; BABIN, B.; MONEY, A. H.; SAMOUEL, P. Fundamentos de métodos de pesquisa em administração. Porto Alegre: Bookman, 2005.

HUTCHESON, G. D.; SOFRONIOU, N. The multivariate social scientist: introductory statistics using generalized linear models. Sage, 1999.

JAQUES, E. Taking time seriously in evaluating jobs. Harvard Business Review, v. 57, n. 5, p. 124-132, 1979.

LE BOTERF, G. Desenvolvendo a competência dos profissionais. Porto Alegre: Artmed, 2003.

LEE, N.; HOOLEY, G. The evolution of "classical mythology" within marketing measure development. European Journal of Marketing, v. 39, n. 3/4, p. 365-385, 2005.

LUZ, T. R. da. Telemar-Minas: competências que marcam a diferença. 2001. Tese (Doutorado em Administração) - Faculdade de Administração e Ciências Econômicas, Universidade Federal de Minas Gerais, Belo Horizonte, 2013.

MALHOTRA, N. K. Pesquisa de Marketing: uma orientação aplicada. 4. ed. Porto Alegre: Bookman, 2006.

MARCONI, M. de A.; LAKATOS, E. M. Fundamentos da metodologia científica. 5 ed. São Paulo: Atlas, 2003.

MEGIDO, J. L. T; XAVIER, C. Marketing \& Agribusiness. 4. ed. São Paulo: Atlas, 2003.

PAIVA, K. C. M. Gestão de competências e a profissão docente um estudo de caso em universidades no Estado de Minas Gerais. 2007. Tese (Doutorado em Administração), Faculdade de Ciências Econômicas, Universidade Federal de Minas Gerais, Belo Horizonte, 2007.

PAIVA, K. C. M.; BARROS, V. R. F.; MENDONÇA, J. R. C.; SANTOS, A. O.; DUTRA, M. R. S. Competências docentes ideais e reais em educação a distância no curso de administração: um estudo em uma instituição brasileira. Tourism \& Management Studies, v. 10, n. ESPECIAL, p. 121-128, 2014.

PAIVA, K. C. M.; FERREIRA, L. S. Competências Gerenciais na área de Tecnologia de Informação: um estudo com gestores de empresas localizadas no Triângulo Mineiro. Revista Gestão \& Tecnologia, v. 13, n. 1, p. 205-229, 2013.

PASQUALI, L. Análise fatorial para pesquisadores. Brasília: Universidade de Brasília - LapPAM, 2005.

PERSPECTIVAS para o agronegócio em 2015. CEPEA- Centro de Estudos Avançados em Economia Aplicada na Universidade de São Paulo, Piracicaba, 9 dez. 2014. Disponível em: http://www.cepea.esalq.usp.br/comunicacao/Cepea Perspectivas\%20Agroneg2015 relatorio. pdf. Acesso em: 20 maio 2015.

Perspectivas em Gestão \& Conhecimento, João Pessoa, v. 7, n. 1, p. 232-247, jan./jun. 2017. 
PETERSON, R. A. A meta-analysis of Cronbach's coefficient alpha. Journal of Consumer Research, v. 21, p. 381-391, 1994.

QUINN, R. E.; ROBERT, E.; THOMPSON, M. P.; FAERMAN, S. R.; MCGRATH, M. Competências gerenciais: princípios e aplicações. Rio de Janeiro: Elsevier, 2003.

RICHARDSON, R. J. Pesquisa social: métodos e técnicas, 3 ed. São Paulo: Atlas, 1999.

ROESCH, S. M. A. Projetos de estágio e de pesquisa em administração. São Paulo: Atlas, 1999.

RUAS, R. Gestão por competência: uma contribuição à estratégia das organizações. In: RUAS, Roberto et al. Os novos horizontes da gestão: aprendizagem organizacional e competências. Porto Alegre: Bookman, 2005.

SANDBERG, J. Understanding human competence at work: an interpretative approach. Academy of Management Journal, New York, v.43, n.1, p.9-25, 2000.

SANTOS, V. C. B. dos; DOS SANTOS, C. A.; BELLUZZO, R. C. B. A competência em informação em articulação com a inteligência competitiva no apoio ao alinhamento estratégico das informações nas organizações. Perspectivas em Gestão \& Conhecimento, v. 6, n. 1, p. 45-60, 2016.

SILVA, I. F.; BINOTTO, E. O conhecimento e a aprendizagem organizacional no contexto de uma organização rural. Perspectivas em Gestão \& Conhecimento, v. 3, n. 1, p. 132-156, 2013.

SILVA, H. C. H.; BINOTTO, E.; VILPOUX, O. F. Cooperação e compartilhamento de informação entre os atores sociais em um assentamento rural. Perspectivas em Gestão \& Conhecimento, v. 6, n. 1, p. 89-108, 2016.

TABACHNICK, B. G. FIDELL, L. S. Using multivariate statistics. New York: Harper Collins, 1989.

TRIVIÑOS, A. N. S. Introdução à pesquisa em ciências sociais: a pesquisa qualitativa em educação. São Paulo, Atlas, 1987.

WOOD JÚNIOR, T. Gestão empresarial: o fator humano. São Paulo: Atlas, 2002.

ZARIFIAN, P. Objetivo competência. São Paulo: Atlas, 2001.

ZUCCARI, P.; BELLUZZO, R. C. B. A competência em informação e o perfil empreendedor no âmbito das organizações. Perspectivas em Gestão \& Conhecimento, v. 6, n. 1, p. 61-71, 2016.

Artigo recebido em 27/02/2016 e aceito para publicação em 15/09/2016 